[Article]

www.whxb.pku.edu.cn

\title{
6-N,N-二甲基腺嘌呤激发态结构动力学的共振拉曼光谱
}

\author{
刘崇杜荵赵彦英王惠钢郑旭明“
}

(浙江理工大学化学系, 先进纺织材料与加工技术教育部重点实验室, 生态染整技术教育部工程研究中心, 杭州 310018)

\begin{abstract}
摘要: 采用共振拉曼光谱技术和密度泛函理论方法研究了 $6-N, N$-二甲基腺嘌呤(DMA)的 $A$ 带和 $B$ 带电子激 发和 Franck-Condon 区域结构动力学. $\Pi_{\mathrm{H}} \rightarrow \Pi_{\mathrm{L}}^{*}$ 跃迁是 $A$ 带吸收的主体, 其振子强度约占整个 $A$ 带吸收的 $79 \%$. 由弥散轨道参与的 $n \rightarrow R y d$ 和 $\pi_{\mathrm{H}} \rightarrow \mathrm{Ryd}$ 跃迁在 $B$ 带跃迁中扮演重要角色, 其振子强度约占 $B$ 带吸收的 $62 \%$, 而 在 $A$ 带吸收中占主导的 $\pi_{\mathrm{H}} \rightarrow \pi_{\mathrm{L}}^{*}$ 跃迁的振子强度在 $B$ 带吸收中仅占 $33 \%$. 嘌呤环变形伸缩 $+\mathrm{C} 8 \mathrm{H} / \mathrm{N} 9 \mathrm{H}$ 面内弯曲 振动 $V_{23}$ 和五元环变形伸缩 $+\mathrm{C} 8 \mathrm{H}$ 弯曲振动 $V_{13}$ 的基频、泛频和合频占据了 $A$ 带共振拉曼光谱强度的绝大部分, 说 明 ${ }^{1} \pi \mathrm{H} \Pi$ 激发态结构动力学主要沿嘌呤环的变形伸缩振动, $\mathrm{N} 9 \mathrm{H} / \mathrm{C} 8 \mathrm{H} / \mathrm{C} 2 \mathrm{H}$ 弯曲振动等反应坐标展开, 而 $V_{10}, V_{29}$, $V_{21}, V_{26}$ 和 $v_{40}$ 的基频、泛频和合频占据了 $B$ 带共振拉曼光谱强度的主体部分, 它们决定了 $B$ 带激发态的结构动力 学. $A$ 带共振拉曼光谱中 $v_{26}$ 和 $v_{12}$ 被认为与 ${ }^{1} n \pi^{*} /{ }^{1} \pi \pi^{\prime \prime}$ 势能面雉型交叉有关. $B$ 带共振拉曼光谱中 $v_{21}$ 的激活与 ${ }^{1} n \pi{ }^{\prime \prime} /$ ${ }^{1} \pi \sigma_{\mathrm{N} H}^{*}$ 势能面雉型交叉相关.
\end{abstract}

关键词： 6- $N, N-$ 二甲基腺嘌呤; 激发态结构动力学; 共振拉曼光谱; 电子激发 中图分类号: 0643

\section{Resonance Raman Spectroscopy of the Excited State Structural Dynamics of 6-N,N-Dimethyladenine}

\author{
LIU Chong DU Rui ZHAO Yan-Ying WANG Hui-Gang ZHENG Xu-Ming*
}

(Key Laboratory of Advanced Textiles Materials and Manufacture Technology, and Engineering Research Center for Eco-dyeing and Finishing of Textiles, Ministry of Education, Department of Chemistry, Zhejiang Science and Technology University, Hangzhou 310018, P. R. China)

\begin{abstract}
The A- and $B$-band electronic excitations and the excited state structural dynamics of 6- $N$, $N$-dimethyladenine (DMA) were studied by resonance Raman spectroscopy and density functional theory calculations. The $\pi_{\mathrm{H}} \rightarrow \pi_{\mathrm{L}}^{*}$ transition is the main part of the A-band absorption and its calculated oscillator strength occupies $79 \%$ of the $A$-band absorption. $n \rightarrow$ Ryd and $\pi_{\mathrm{H}} \rightarrow$ Ryd transitions where Ryd denotes the diffuse Rydberg orbital play important roles in the $B$-band electronic transitions and their calculated oscillator strengths occupy about $62 \%$ of the $B$-band absorption. The oscillator strength for the $\pi_{\mathrm{H}} \rightarrow \pi_{\llcorner}^{*}$ transition, which dominates the $A$-band electronic transition only occupies about $33 \%$ of the $B$-band absorption. The foundamental vibrations of the purine ring deformation stretch plus the $\mathrm{C} 8 \mathrm{H} / \mathrm{N} 9 \mathrm{H}$ bend mode $v_{23}$ and the 5 member ring deformation stretch plus the $\mathrm{C} 8 \mathrm{H}$ bend mode $v_{13}$, and their overtones and combination bands occupy most of the $A$-band resonance Raman intensities. Therefore, the ${ }^{1} \pi_{H} \pi_{L}^{*}$ excited state structural dynamics of DMA is mainly along the $v_{23}$ and $v_{13}$ reaction coordinates. The majority of the $B$-band resonance Raman intensities are dominated by the fundamental vibrations of $v_{10}, v_{29}, v_{21}, v_{26}, v_{40}$, and their overtones and combination bands. This suggests the $B$-band excited state structural dynamics of DMA is mostly along the purine ring deformation, the $\mathrm{C} 6 \mathrm{~N} 10$ stretch, the $\mathrm{N} 9 \mathrm{H} / \mathrm{C} 8 \mathrm{H} / \mathrm{C} 2 \mathrm{H}$ bend and the
\end{abstract}

Received: August 13, 2010; Revised: October 13, 2010; Published on Web: December 13, 2010.

"Corresponding author. Email: zxm@zstu.edu.cn; Tel: +86-571-86843699.

The project was supported by the National Key Basic Research Program of China (973) (2007CB815203), National Natural Science Foundation of China (21033002, 20573097), and Natural Science Foundation of Zhejiang Province, China (R405465).

国家重点基础研究发展规划(973) (2007CB815203), 国家自然科学基金(21033002, 20573097)和浙江省自然科学基金(R405465)资助项目

(C) Editorial office of Acta Physico-Chimica Sinica 
$\mathrm{N}\left(\mathrm{CH}_{3}\right)_{2}$ antisymmetric stretch. The appearance of $V_{26}$ and $v_{12}$ in the A-band resonance Raman spectrum is correlated to the Franck-Condon region ${ }^{1} n \pi^{*} /{ }^{1} \pi \pi^{*}$ conical intersection. The activation of $v_{21}$ in the $B$-band resonance Raman spectrum is correlated to the Franck-Condon region ${ }^{1} \pi \pi^{*} / 1 \pi \sigma_{\mathrm{N \mu H}}^{*}$ conical intersection.

Key Words: $\quad 6-\mathrm{N}, \mathrm{N}$-dimethyladenine; Excited state structural dynamics; Resonance Raman spectrum; Electronic transition

核酸光稳定性与核酸碱基激发态超快内转换 过程密切相关 ${ }^{[1]}$. 在腺嘌呤光稳定机制的理论和光 谱实验研究中, Domcke ${ }^{[2]}$ 和 Sobolewski 等 ${ }^{[3-4]}$ 首先提 出 ${ }^{1} \sigma \pi^{*}$ 激发态在吡咯等杂芳烃激发态势能面交叉和 超快内转换中的重要性. 随后, 用时间分辨质谱

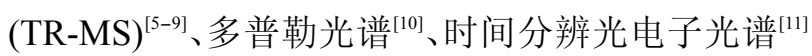
和其他手段 ${ }^{[12]}$ 发现和证实了激发态腺嘌呤的 $\mathrm{H}$ - 原 子消除反应及其与激光波长的依赖关系.Wells 等 ${ }^{[8-9]}$ 注意到, 采用 $200 \mathrm{~nm}$ ( $B$ 吸收带) 光激发时, ${ }^{1} \sigma \pi^{*} /{ }^{1} \pi \pi^{*}$ 势能面锥形交叉成为腺嘌呤激发态能量衰减的一 条重要通道, 而采用 $266 \mathrm{~nm}$ ( $A$ 吸收带)光激发时, 这 一通道的作用则很小. 通过 $\mathrm{H}$-原子消除反应的时间 响应函数拟合, 腺嘌呤显示 $\tau_{1}=(110 \pm 63) \mathrm{fs}$ 和 $\tau_{2}=$ (265 135$)$ fs 两个时间常数, 而 6- $N, N-$ 二甲基腺嘌呤 (DMA) 仅显示 $\tau_{1}=(95 \pm 21) \mathrm{fs}$ 一个时间常数. 这些实 验研究明确了激发态 ${ }^{1} \sigma \pi^{*} /{ }^{1} \pi \pi^{*}$ 势能面雉形交叉在腺 嘌呤光化学中的作用, 确认了腺嘌呤 N9 和 N6 原子 上两个 $\mathrm{H}$-原子消除反应通道. 飞秒时间分辨光谱研 究同时揭示, 腺嘌呤在激发态发生内转换过程的时 间尺度约为皮秒-亚皮秒量级 ${ }^{[6-9,12-15]}$.

腺嘌呤有 $9 \mathrm{H}$-和 $7 \mathrm{H}$-两种异构体, 基态时以 $9 \mathrm{H}$ 形式存在 ${ }^{[16-17]}$, 而荧光发射体为 $7 \mathrm{H}$ - 异构体 ${ }^{[17-18]}$. DMA 也存在 $9 \mathrm{H}$-和 $7 \mathrm{H}$-异构体, 但 $7 \mathrm{H}$-DMA 含量要 比 $9 H-D M A$ 少得多 ${ }^{[19]}$. Parusel 等 ${ }^{[19]}$ 的研究结果表 明, 与腺嘌呤 $7 \mathrm{H}$ 异构体发射单一的苂光光谱带不 同, DMA 的 $9 \mathrm{H}$ 异构体在不同极性的溶剂中发射双 荧光, 即 $A$ 带和 $B$ 带苂光. $A$ 带荧光能量较低, 由扭曲 的分子内电荷转移(TICT)态的辐射失活引发, $B$ 带 苂光能量较高, 由嘌呤环的局域激发态的发射引起. 显然, 环外氨基的 $N, N$-二甲基化改变了腺嘌呤的激 发态结构动力学和荧光发射过程. 最近, Schwalb 和 $\mathrm{Temps}^{[20]}$ 采用飞秒时间分辨苂光光谱实验, 对 DMA 的 $A$ 带苂光动力学机制提出了新解释, 把 $A$ 带苂光 归属于 ${ }^{1} n \pi^{*}$ 态的发射.

DMA 激发态动态结构研究至今未见报道. 原 理上, 振动分辨的电子光谱学能提供分子激发态结
构动力学的具体信息, 但在凝聚态和超短激发态寿 命等条件下, 光谱展宽使得这一目的难以实现. 共 振拉曼光谱学作为激发态结构动力学研究的强有 力探针技术, 通过获取反应坐标信息, 可以在研究 Franck-Condon(FC) 区域势能面交叉和电子态耦合 等方面发挥独特的作用. 因此, 本文采用共振拉曼 光谱技术, 研究 DMA 在水和乙腈中 $A$ 带和 $B$ 带电子 激发条件下的 $\mathrm{FC}$ 区域结构动力学, 考察环外氨基 的甲基化对腺嘌呤不同激发态结构动力学或反应 坐标的影响, 并以此为依据洞察势能面交叉和电子 态耦合信息.

\section{1 实验部分}

\section{1 试剂和仪器}

DMA(纯度 $98 \%$, Toronto Research Chemical 公 司). 乙腈试剂(光谱纯, 纯度为 $99.9 \%$, 安徽时联特种 试剂股份有限公司).

\section{2 实验方法}

分别用乙腈和水做溶剂, DMA 的浓度约为 $4.00 \times 10^{-3} \mathrm{~mol} \cdot \mathrm{L}^{-1}$. 共振拉曼光谱实验装置和方法参 照文献[21]. 由 Nd:YAG 纳秒脉冲激光器产生 532.0、 354.7 和 $266.0 \mathrm{~nm}$ 激光, 它们经氢气受激拉曼位移管 产生 282.4、273.9、266.0、239.5、217.8、208.8 nm 激 光. 采用流动循环方式进样. 拉曼散射信号采用背 向散射几何结构, 经椭球镜等聚焦于单色仪的入口 狭缝, 由光栅分光后进入液氮冷却的电荷-耦合装置 (CCD) 检测器搜集. 信号经多次(20-30个)累加, 最 后得到拉曼光谱. 共振拉曼光谱的振动频率通过与 乙腈拉曼谱带的已知频率值比较进行校正. 从样品 溶液的共振拉曼光谱减去纯乙腈溶剂(或水)的拉曼 光谱, 得到 6 个激发波长下 DMA 分别在乙腈和水溶 剂中的共振拉曼光谱. 其中, 共振拉曼光谱的自吸 收校正和实验装置中信号收集系统的强度校正原 理见文献[22], 具体校正通过 Origin 软件的自编程 序完成. 


\section{2 理论计算}

电子基态几何结构和简正振动频率用密度泛 函理论在 B3LYP/6-31++ $\mathrm{G}(d f, p d)$ 水平计算获得. 电 子跃迁能在 B3LYP-TD/6-31++ $\mathrm{G}(d f, p d)$ 水平计算得 到. 本文所有量子化学计算均由 Gaussian $03 \mathrm{~W}$ 程序 包 ${ }^{[23]}$ 完成.

\section{3 结果和讨论}

\section{1 基态几何结构}

图 1 为 $9 \mathrm{H}-\mathrm{DMA}$ 的几何结构. Parusel 等 ${ }^{[19]}$ 利用 AM1 半经验量子力学方法对 $9 \mathrm{H}$-和 7H-异构体的基 态结构进行了优化计算. 结果表明, 常温下 $9 \mathrm{H}$-异构 体与 $7 \mathrm{H}$-异构体的能差为 $17.6 \mathrm{~kJ} \cdot \mathrm{mol}^{-1}, 9 \mathrm{H}$-异构体 占据绝对优势 (分子数比 1000:1). 本文在 B3LYP/ 6-31++g $(d f, p d)$ 计算水平下获得的 $9 \mathrm{H}$-和 $7 \mathrm{H}$-异构体 的能差为 $37.4 \mathrm{~kJ} \cdot \mathrm{mol}^{-1}$ (零点能校正后为 $34.4 \mathrm{~kJ}$. $\mathrm{mol}^{-1}$ ), 与 $\mathrm{AM} 1$ 结果定性一致, 但能差进一步拉大, 说明基态时 DMA 以 9H-异构体形式存在. 9H-DMA 两甲基之间的相对扭转产生构象异构体, 主要有交 叉式和重叠式两种构象. 在 B3LYP/6-31++G $(d f, p d)$ 计算水平下, 交叉式构象为鞍点结构, 重叠式构象 为能量最低结构. 重叠式构象可进一步细分为平面 结构和雉形化结构. 锥形化结构起因于二甲基氨基 中氮原子对嘌呤环平面的偏离. 结构优化和频率计 算显示平面分子为鞍点结构, 而非平面分子较平面 分子的能量低 $0.185 \mathrm{~kJ} \cdot \mathrm{mol}^{-1}$, 扭转角 (与平面分子 的二面角之差值)为 $7.8^{\circ}-11.1^{\circ}$, 表明非平面的重叠 式构象为能量最低结构. 这一结果与二甲基苯胺中 氨基约 $7.5^{\circ}$ 的非平面角相似 ${ }^{[24]}$.

\section{2 振动光谱}

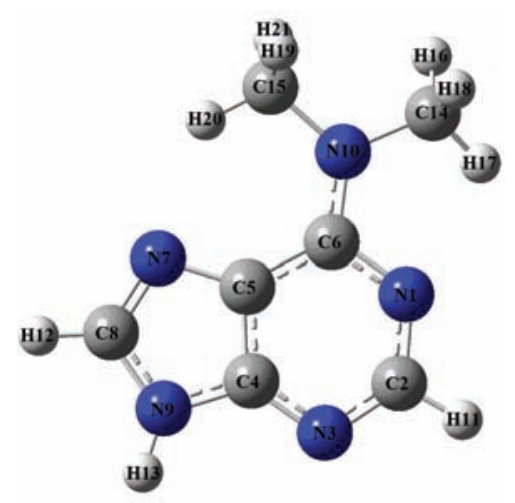

图 1 9H-DMA 的几何结构示意图

Fig.1 Schematic diagram of the geometry structure of 9H-DMA
DMA 振动光谱指认尚无文献报导. 为了指认 共振拉曼光谱, 对 DMA 的 $9 \mathrm{H}$-和 $7 \mathrm{H}$-构象异构体的 拉曼光谱进行了计算研究. 图 2 显示 B3LYP/6-31++ $\mathrm{G}(d f, p d)$ 计算获得的 9H-DMA 和 7H-DMA 异构体拉 曼光谱, $\mathrm{a}$ 和 $\mathrm{b}$ 谱分别表示 $9 \mathrm{H}$-异构体的重叠构象和 交叉构象, $\mathrm{c}$ 和 $\mathrm{d}$ 谱表示 $7 \mathrm{H}$-异构体的重叠构象和交 叉构象. $\mathrm{b}$ 和 $\mathrm{c}$ 谱表示各有一个虚频, 表明是鞍点结 构的振动光谱. $\mathrm{a}$ 和 $\mathrm{d}$ 谱分别为 $9 \mathrm{H}$-异构体非平面重 叠构象和 $7 \mathrm{H}$-异构体非平面交叉构象的拉曼光谱. 由图 2 可见,在 1250-1700 $\mathrm{cm}^{-1}$ 区域, 9H-DMA 和 $7 \mathrm{H}-\mathrm{DMA}$ 拉曼光谱的强度模式差别很大, 它们当中 $a$ 光谱与实验拉曼光谱最吻合. 这从拉曼光谱的角 度证明 DMA 主要以 $9 \mathrm{H}$-异构体形式存在, 与 $\mathrm{AM} 1$ 计算 ${ }^{[19]}$ 和本文 $\mathrm{B} 3 \mathrm{LYP} / 6-31++\mathrm{G}(d f, p d)$ 计算预示的稳 定性结果一致. 在计算的 $9 \mathrm{H}-\mathrm{DMA}$ 重叠构象拉曼光 谱中, 1400-1550 $\mathrm{cm}^{-1}$ 区域的强度模式受基组的影 响比较明显. 结果表明, 在所选的 6-31G $(d) 、 6-31+$ $\mathrm{G}(d, p) 、 6-311+\mathrm{G}(2 d f, p) 、 6-31++\mathrm{G}(d f, p d) 、 6-311++$ $\mathrm{G}(3 d f, 3 p d) 5$ 个基组中, 除 6-31G $(d)$ 基组外, 其它基 组都能较好地重现实验结果. 其中, 后三个基组的 计算光谱差别甚微, 这表明对9H-DMA 重叠构象采 用 B3LYP/6-31++ G $(d f, p d)$ 计算水平既可以获得与 实验拉曼光谱相吻合的计算拉曼光谱, 又可以节省 计算成本. 表 1 列出了 $9 \mathrm{H}-\mathrm{DMA}$ 非平面重叠构象的

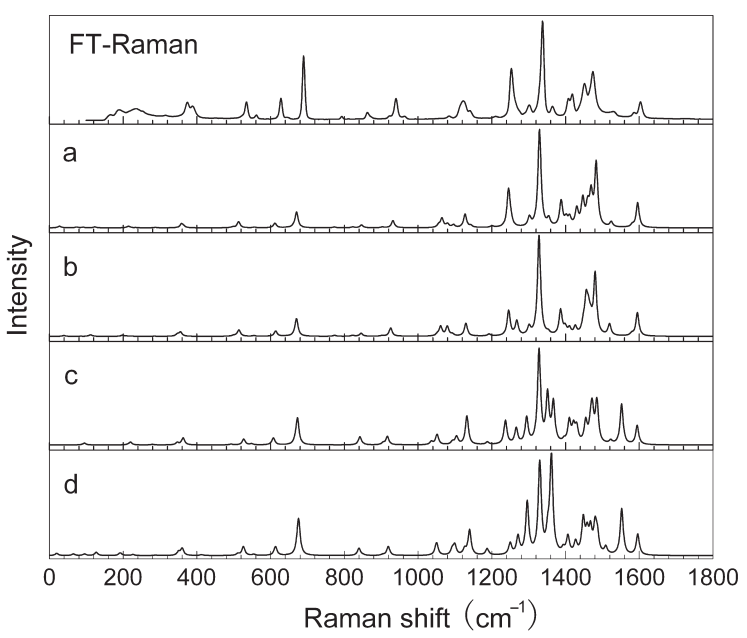

图 2 实验拉曼光谱(FT-Raman)与计算拉曼 光谱 $(\mathbf{a}, \mathbf{b}, \mathbf{c}, \mathbf{d})$ 比较

Fig.2 The comparison of the experimental Raman spectrum (FT-Raman) to the calculated Raman spectra (a, b, c, d)

(a) and (b) are the eclipsed conformation and the staggered conformation of 9H-DMA, respectively. (c) and (d) are eclipsed conformation and staggered conformation of 7H-DMA, respectively. 
表 1 9H-DMA 理论振动频率和实验谱带的指认

Table 1 Assignment of theoretical vibrational frequencies and experimental bands of 9H-DMA

\begin{tabular}{|c|c|c|c|c|c|}
\hline \multirow{2}{*}{ Mode } & \multicolumn{2}{|c|}{ Calc. } & \multicolumn{2}{|c|}{ Expt. } & \multirow{2}{*}{ Assignment } \\
\hline & $\mathrm{a}$ & $\mathrm{b}$ & FT-Raman & R.Raman & \\
\hline$v_{1}$ & 3668 & 3572 & & & N9H stretch \\
\hline$v_{2}$ & 3253 & 3169 & & & $\mathrm{C} 8 \mathrm{H}$ stretch \\
\hline$v_{3}$ & 3173 & 3092 & & & $\mathrm{C} 2 \mathrm{H}$ stretch \\
\hline$v_{4}$ & 3172 & 3091 & & & $\mathrm{C} 15 \mathrm{H} 20 / \mathrm{C} 14 \mathrm{H} 17 / \mathrm{C} 2 \mathrm{H}$ stretch \\
\hline$v_{5}$ & 3169 & 3088 & & & $\mathrm{C} 14 \mathrm{H} 17 / \mathrm{C} 15 \mathrm{H} 20 / \mathrm{C} 2 \mathrm{H}$ stretch \\
\hline$v_{6}$ & 3066 & 2988 & & & $\mathrm{H} 16 \mathrm{C} 14 \mathrm{H} 18$ asym stretch \\
\hline$v_{7}$ & 3050 & 2973 & & & H19C15H21 asym stretch \\
\hline$v_{8}$ & 2998 & 2922 & & & H19C15/C14H18 stretch \\
\hline$v_{9}$ & 2991 & 2915 & & & $\mathrm{CH}_{3}$ stretch \\
\hline$v_{10}$ & 1632 & 1597 & $1603 \mathrm{~m}$ & 1594 & $\mathrm{PR}$ deform $+\mathrm{C} 6 \mathrm{~N} 10$ stretch $+\mathrm{N} 9 \mathrm{H} / \mathrm{C} 2 \mathrm{H}$ bend $+\mathrm{CH}_{3}$ scissor \\
\hline$v_{11}$ & 1617 & 1582 & $1586 w$ & & $\mathrm{PR}$ deform $+\mathrm{C} 6 \mathrm{~N} 10$ stretch $+\mathrm{N} 9 \mathrm{H} / \mathrm{C} 8 \mathrm{H}$ bend $+\mathrm{CH}_{3}$ scissor \\
\hline$v_{12}$ & 1559 & 1526 & $1529 w$ & 1545 & $\mathrm{C} 6 \mathrm{~N} 10$ stretch $+\mathrm{CH}_{3}$ scissor $+\mathrm{PR}$ deform $+\mathrm{C} 2 \mathrm{H} / \mathrm{C} 8 \mathrm{H}$ bend \\
\hline$v_{13}$ & 1518 & 1486 & $1474 \mathrm{~s}$ & 1489 & $\mathrm{R} 5$ deform $+\mathrm{C} 8 \mathrm{H}$ bend $+\mathrm{CH}_{3}$ rock \\
\hline$v_{14}$ & 1505 & 1474 & & & $\mathrm{CH}_{3}(\mathrm{C} 15)$ twist \\
\hline$v_{15}$ & 1504 & 1473 & & & $\mathrm{CH}_{3}(\mathrm{C} 15)$ twist \\
\hline$v_{16}$ & 1494 & 1463 & & & $\mathrm{CH}_{3}(\mathrm{C} 14)$ twist \\
\hline$v_{17}$ & 1481 & 1451 & & & $\mathrm{CH}_{3}(\mathrm{C} 14)$ twist \\
\hline$v_{18}$ & 1464 & 1434 & & & $\mathrm{CH}_{3}(\mathrm{C} 15)$ umbrella $+\mathrm{C} 6 \mathrm{~N} 10$ stretch \\
\hline$v_{19}$ & 1445 & 1416 & & & $\mathrm{PR}$ deform $(\mathrm{C} 4)+\mathrm{C} 2 \mathrm{H}$ bend \\
\hline$v_{20}$ & 1435 & 1406 & & & $\mathrm{CH}_{3}(\mathrm{C} 14)$ umbrella \\
\hline$v_{21}$ & 1421 & 1392 & & 1432 & $\mathrm{~N} 9 \mathrm{H} / \mathrm{C} 2 \mathrm{H}$ bend + PR deform(N1,N9) \\
\hline$v_{22}$ & 1387 & 1359 & $1364 w$ & 1376 & $\mathrm{C} 2 \mathrm{H}$ bend $+\mathrm{PR}$ deform \\
\hline$v_{23}$ & 1362 & 1335 & $1337 \mathrm{vs}$ & 1343 & $\mathrm{PR}$ deform $+\mathrm{C} 8 \mathrm{H} / \mathrm{N} 9 \mathrm{H}$ bend \\
\hline$v_{24}$ & 1334 & 1308 & $1302 \mathrm{w}$ & & $\mathrm{PR}$ deform $+\mathrm{C} 8 \mathrm{H}$ bend \\
\hline$v_{25}$ & 1283 & 1259 & & & $\mathrm{~N} 1 \mathrm{C} 6 / \mathrm{C} 14 \mathrm{~N} 10$ stretch $+\mathrm{CH}_{3}(\mathrm{C} 15)$ rock $+\mathrm{N} 9 \mathrm{H} / \mathrm{C} 8 \mathrm{H} / \mathrm{C} 2 \mathrm{H}$ bend \\
\hline$v_{26}$ & 1277 & 1253 & $1253 \mathrm{~s}$ & 1266 & $\mathrm{PR}$ deform $+\mathrm{N}\left(\mathrm{CH}_{3}\right)_{2}$ asym stretch $+\mathrm{CH}_{3}$ rock $+\mathrm{N} 9 \mathrm{H} / \mathrm{C} 8 \mathrm{H}$ bend \\
\hline$v_{27}$ & 1231 & 1208 & $1211 \mathrm{w}$ & 1224 & $\mathrm{PR}$ deform $+\mathrm{CH}_{3}$ rock $+\mathrm{C} 8 \mathrm{H}$ bend \\
\hline$v_{28}$ & 1173 & 1152 & & & $\mathrm{CH}_{3}$ wag \\
\hline$v_{29}$ & 1156 & 1135 & & 1143 & $\mathrm{PR}$ deform $+\mathrm{N}\left(\mathrm{CH}_{3}\right)_{2}$ asym stretch $+\mathrm{C} 8 \mathrm{H}$ bend \\
\hline$v_{30}$ & 1125 & 1105 & & & $\mathrm{CH}_{3}$ twist \\
\hline$v_{31}$ & 1108 & 1089 & & & $\mathrm{CH}_{3}(\mathrm{C} 15)$ rock $+\mathrm{C} 4 \mathrm{~N} 9$ stretch $+\mathrm{N} 9 \mathrm{H} / \mathrm{C} 2 \mathrm{H}$ bend \\
\hline$v_{32}$ & 1093 & 1074 & & 1097 & $\mathrm{C} 8 \mathrm{~N} 9$ stretch $+\mathrm{N} 9 \mathrm{H} / \mathrm{C} 2 \mathrm{H}$ bend \\
\hline$v_{33}$ & 1084 & 1065 & & & $\mathrm{CH}_{3}$ rock \\
\hline$v_{34}$ & 981 & 966 & & & C2Hout-plane wag \\
\hline$v_{35}$ & 958 & 943 & $940 \mathrm{~m}$ & 957 & PR deform $(\mathrm{N} 7, \mathrm{~N} 9)+\mathrm{N}\left(\mathrm{CH}_{3}\right)_{2}$ sym stretch \\
\hline$v_{36}$ & 929 & 915 & & & PR deform $(\mathrm{N} 1, \mathrm{~N} 7)+\mathrm{N}\left(\mathrm{CH}_{3}\right)_{2}$ sym stretch $+\mathrm{C} 8 \mathrm{H}$ bend \\
\hline$v_{37}$ & 872 & 860 & & 880 & PR deform $+\mathrm{N}\left(\mathrm{CH}_{3}\right)_{2}$ sym stretch \\
\hline$v_{38}$ & 848 & 837 & & & C8Hout-plane wag \\
\hline$v_{39}$ & 797 & 787 & & & PR out-plane deform $+\mathrm{C} 8 \mathrm{H}$ out-plane wag \\
\hline$v_{40}$ & 693 & 686 & $689 \mathrm{~s}$ & 701 & purine ring deform \\
\hline$v_{41}$ & 683 & 677 & & & PR out-plane deform $(\mathrm{C} 4, \mathrm{C} 6)$ \\
\hline$v_{42}$ & 666 & 660 & & & R5 out-plane wag N9H/C8H out-plane wag \\
\hline$v_{43}$ & 633 & 628 & $628 \mathrm{~m}$ & 642 & purine ring deform \\
\hline$v_{44}$ & 577 & 574 & & & $\mathrm{~N} 9 \mathrm{H} / \mathrm{C} 2 \mathrm{H}$ out-plane wag $+\mathrm{C} 5$ centered umbrella \\
\hline$v_{45}$ & 534 & 532 & & & N9H/C2H out-plane wag \\
\hline$v_{46}$ & 533 & 531 & $534 \mathrm{~m}$ & 545 & R6 deform $+\mathrm{N} 9 \mathrm{H}$ out-plane wag \\
\hline$v_{47}$ & 516 & 515 & & & $\mathrm{~N}\left(\mathrm{CH}_{3}\right)_{2}$ scissor+R6 breath \\
\hline$v_{48}$ & 382 & 385 & $389 \mathrm{~m}$ & & $(\mathrm{C} 15) \mathrm{CH}_{3}$ bend $+\mathrm{N} 7 \mathrm{C} 5 \mathrm{C} 6$ scissor \\
\hline$v_{49}$ & 376 & 379 & $374 \mathrm{~m}$ & & $(\mathrm{C} 14) \mathrm{CH}_{3}$ bend \\
\hline$v_{50}$ & 304 & 309 & & & $\mathrm{~N}\left(\mathrm{CH}_{3}\right)_{2}$ wag+PR butterfly \\
\hline$v_{51}$ & 250 & 257 & & & $\mathrm{~N}\left(\mathrm{CH}_{3}\right)_{2}$ wag \\
\hline$v_{52}$ & 231 & 238 & $235 w$ & 245 & $\mathrm{~N}\left(\mathrm{CH}_{3}\right)_{2}$ rock \\
\hline$v_{53}$ & 227 & 234 & & & butterfly \\
\hline$v_{54}$ & 137 & 147 & & & $(\mathrm{C} 15) \mathrm{CH}_{3}$ twist $+\mathrm{PR}$ out-plane deform \\
\hline$v_{55}$ & 105 & 116 & & & $\mathrm{CH}_{3}$ twist \\
\hline$v_{56}$ & 87 & 98 & & & $\mathrm{CH}_{3}$ twist \\
\hline$v_{57}$ & 40 & 53 & & & $\mathrm{CH}_{3}$ twist \\
\hline
\end{tabular}

a) B3LYP/6-31++G( $d f, p d)$ calculated, b) scaled = calc. $\times 0.985-12$ (method see Ref.[25]);

R6: six-membered ring; R5: five-membered ring, sym: symmetrical, asym: antisymmetrical,

PR: purine ring, vs: very strong, s: strong, m: middle, w: weak 
实验(FT-Raman 和 R. Raman)和计算所得振动频率 及其光谱指认. 表 1 中 $\mathrm{b}$ 列数据是由 Rauhut 的线性 标度法, 通过 FT-Raman 光谱实验值对计算频率 (a 列)进行校正获得的 ${ }^{[25]}$.

\section{3 电子光谱}

图 3 为 9H-DMA 在水和乙腈溶剂中的紫外吸收 光谱. $208.8 、 217.8 、 239.5 、 266.0 、 273.9$ 和 $282.4 \mathrm{~nm}$ 为 共振拉曼实验所用的激光波长. 图 3 中最大吸收波 长为 275 和 $212 \mathrm{~nm}$ 的两个强吸收峰, 分别被称为 $A$ 带和 $B$ 带吸收. 质子性溶剂(水)和非质子性溶剂(乙 腈)对紫外光谱的吸收强度和谱峰位移影响不大. 表 2 列出在 B3LYP-TD $/ 6-31++\mathrm{G}(d f, p d)$ 计算水平下 9H-DMA 的电子跃迁能 $(\Delta E)$ 和振子强度 $(f)$. 由表 2 可见, 在 200-400 nm 紫外光谱区域, 计算给出 8 个 振子强度 $f>0.01$ 的电子跃迁带, 其中 266-249 nm 吸收带 $(A$ 带)和 212-199 nm 吸收带 $(B$ 带)的振子强 度分别为 $\Sigma f_{i}=0.33$ 和 $\Sigma f_{i}=0.30$, 分别略低于对应的实 验值 $f=0.37$ 和 $f=0.33$. 溶剂效应对振子强度稍有影 响, 采用 SCIPCM 溶剂模型获得的 $A$ 带和 $B$ 带的振 子强度分别为 $\Sigma f_{i}=0.39$ 和 $\Sigma f_{i}=0.36$, 分别略高于实验 值.

为了便于理解激发态态的电子结构, 有必要对 前线轨道的特征做一简要讨论. 这里, 我们借用了 Marian 等 ${ }^{[26}$ 对弥散轨道的描述. 最高占有分子轨道 (HOMO) 和第三最高占有分子轨道(HOMO-2) 是 $\pi$ 成键轨道, 分别表示为 $\pi_{\mathrm{H}}$ 和 $\pi_{\mathrm{H}-2}$. 第二最高占有分子 轨道(HOMO-1) 是面内非键轨道 $n$, 其主要特征为 N1、N3 和 N7 的孤对电子轨道. 最低空价键轨道

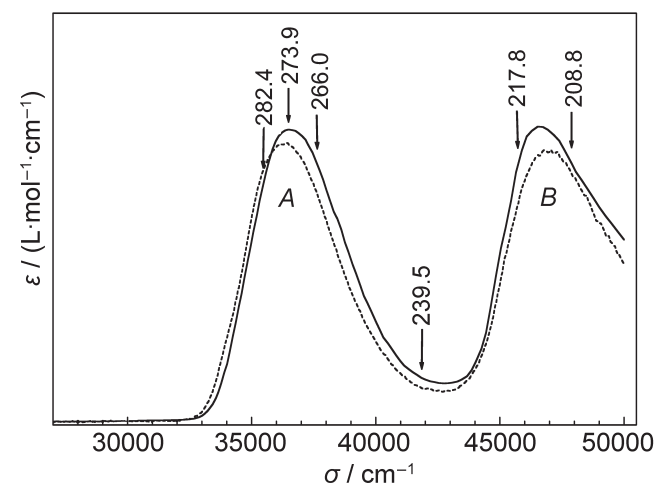

图 $39 \mathrm{H}-\mathrm{DMA}$ 在水(虚线)和乙腈(实线)溶剂中的 紫外吸收光谱

Fig.3 Absorption spectra of 9H-DMA in water (dashed line) and acetonitrile (solid line) solvents

The excitation wavelengths used for the resonance Raman experiments are indicated above the absorption spectra.
(LUMO) 和第二最低空价键轨道 $(\mathrm{LUMO}+1)$ 是 $\pi^{*}$ 反 键轨道 $\left(\pi_{\mathrm{L}}^{*}\right.$ 和 $\left.\pi_{\mathrm{L}+1}^{*}\right)$. 在 $\pi_{\mathrm{L}}^{*}$ 和 $\pi_{\mathrm{L}+1}^{*}$ 能隙之间, 有四个弥散 轨道. 第一、三弥散轨道 $\left(\mathrm{Ryd}_{1}\right)$ 的密度涵盖 $\mathrm{H} 13$ 和 $\mathrm{H} 12$ 的环外区域, 第二、四弥散轨道 $\left(\mathrm{Ryd}_{2}\right.$ 和 $\left.\mathrm{Ryd}_{4}\right)$ 的 密度包含 $\mathrm{N}\left(\mathrm{CH}_{3}\right)_{2}$ 基团四个面外 $\mathrm{H}$ 原子的区域, 而第 五弥散轨道 $\left(\mathrm{Ryd}_{5}\right)$ 的密度包含嘌呤环上下空间和 $\mathrm{H} 11$ 和 H19,20,21 的外部区域.

轨道系数和振子强度数据表明, 在真空条件 下, $\pi_{\mathrm{H}} \rightarrow \pi_{\mathrm{L}}^{*}$ 跃迁是 $A$ 带吸收的主体, 其振子强度占整 个 $A$ 带吸收的计算振子强度的 $79 \%, \pi_{\mathrm{H}} \rightarrow \mathrm{Ryd}_{2}$ 跃迁 也相当重要, 占总振子强度的 $19 \%$. 而与 N9-H 原子 消除反应通道有关的 $\pi_{\mathrm{H}} \rightarrow \mathrm{Ryd}_{1}$ 跃迁和与 ${ }^{1} n \pi^{*} /{ }^{\prime} \pi^{*}$ 内转 换有关的 $n_{\mathrm{H}-1} \rightarrow \pi_{\mathrm{L}}^{*}$ 跃迁占总振子强度的 $2 \%$, 它们对 $A$ 带吸收作用微小, 但作为暗态或暗结构, 它们可能 在激发态势能面交叉方面发挥重要作用. $B$ 带跃迁 的一个显著特征是弥散轨道扮演重要角色, 概括起 来 $n \rightarrow \operatorname{Ryd}$ 跃迁和 $\pi_{\mathrm{H}} \rightarrow \operatorname{Ryd}$ 跃迁占总振子强度的 $62 \%$, 而在 $A$ 带吸收中占主导的 $\pi \rightarrow \pi^{*}$ 跃迁在 $B$ 带吸 收中仅占 $33 \%$. 剩余的 $5 \%$ 为 $n_{\mathrm{H}-1} \rightarrow \pi_{\mathrm{L}+1}^{*}$ 跃迁吸收. 值 得注意的是, 尽管 $\pi_{\mathrm{H}} \rightarrow \mathrm{Ryd}_{3}$ 跃迁对 $B$ 带电子跃迁吸 收的贡献可以忽略, 但由于弥散轨道 $\mathrm{Ryd}_{3}$ 具有 $\sigma_{\mathrm{N} \text { 斯 }}^{*}$ 特征, 已有研究提出的激发态 N9-H 原子消除反应 的 $^{1} \pi \pi^{*} /{ }^{1} \pi \sigma_{\mathrm{N} \text { H }}^{*}$ 锥形交叉机制可能使得这一跃迁在激

\section{表 2 计算获得的 9H-DMA 的电子跃迁能 $(\Delta E)$ 和 振子强度 $(f)$}

Table 2 Calculated electronic transition energies $(\Delta E)$ and oscillator strengths $(f)$ for $9 \mathrm{H}-\mathrm{DMA}$

\begin{tabular}{|c|c|c|c|c|c|c|}
\hline \multirow{2}{*}{ State } & \multirow{2}{*}{$\begin{array}{c}\text { Orbital } \\
\text { (coefficient) }\end{array}$} & \multirow{2}{*}{ Character } & \multicolumn{2}{|c|}{$\Delta E / \mathrm{nm}$} & \multicolumn{2}{|r|}{$f$} \\
\hline & & & Calc. & Expt. & Calc. & Expt. \\
\hline$\overline{S_{1}}$ & $43 \rightarrow 44(0.62)$ & $\pi_{\mathrm{H}} \rightarrow \pi_{\mathrm{L}}^{*}$ & 266 & \multirow{4}{*}{275} & 0.2606 & \multirow{4}{*}{$\begin{array}{c}0.3727 \\
(A \text { band) }\end{array}$} \\
\hline$S_{2}$ & $43 \rightarrow 45(0.67)$ & $\pi_{\mathrm{H}} \rightarrow \mathrm{Ryd}_{1}$ & 256 & & 0.0026 & \\
\hline$S_{3}$ & $42 \rightarrow 44(0.68)$ & $n_{\mathrm{H}-1} \rightarrow \pi_{\mathrm{L}}^{*}$ & 253 & & 0.0033 & \\
\hline$S_{4}$ & $43 \rightarrow 46(0.55)$ & $\pi_{\mathrm{H}} \rightarrow \mathrm{Ryd}_{2}$ & 249 & & 0.0614 & \\
\hline$S_{10}$ & $43 \rightarrow 49(0.69)$ & $\pi_{\mathrm{H}} \rightarrow \mathrm{Ryd}_{3}$ & 212 & & 0.0034 & 0.3338 \\
\hline$S_{11}$ & $43 \rightarrow 51(0.61)$ & $\pi_{\mathrm{H}} \rightarrow \pi_{\mathrm{L}+2}^{*}$ & 212 & & 0.0392 & ( $B$ band) \\
\hline \multirow[t]{2}{*}{$S_{12}$} & $41 \rightarrow 44(0.31)$ & $\pi_{\mathrm{H}-2} \rightarrow \pi_{\mathrm{L}}^{*}$ & \multirow[t]{2}{*}{209} & & \multirow[t]{2}{*}{0.0428} & \\
\hline & $42 \rightarrow 45(0.49)$ & $n_{\mathrm{H}-1} \rightarrow \mathrm{Ryd}_{1}$ & & & & \\
\hline \multirow[t]{2}{*}{$S_{13}$} & $42 \rightarrow 45(0.42)$ & $n_{\mathrm{H}-1} \rightarrow \mathrm{Ryd}_{1}$ & \multirow[t]{2}{*}{208} & & \multirow[t]{2}{*}{0.0194} & \\
\hline & $43 \rightarrow 52(0.50)$ & $\pi_{\mathrm{H}} \rightarrow \mathrm{Ryd}_{4}$ & & & & \\
\hline \multirow[t]{5}{*}{$S_{14}$} & $42 \rightarrow 46(0.26)$ & $n_{\mathrm{H}-1} \rightarrow \mathrm{Ryd}_{2}$ & \multirow[t]{5}{*}{204} & \multirow[t]{5}{*}{212} & \multirow[t]{5}{*}{0.1076} & \\
\hline & $42 \rightarrow 47(0.13)$ & $n_{\mathrm{H}-1} \rightarrow \pi_{\mathrm{L}+1}^{*}$ & & & & \\
\hline & $43 \rightarrow 47(0.12)$ & $\pi_{\mathrm{H}} \rightarrow \pi_{\mathrm{L}+1}^{*}$ & & & & \\
\hline & $43 \rightarrow 51(0.14)$ & $\pi_{\mathrm{H}} \rightarrow \pi_{\mathrm{L}+2}^{*}$ & & & & \\
\hline & $43 \rightarrow 53(0.35)$ & $\pi_{\mathrm{H}} \rightarrow \mathrm{Ryd}_{5}$ & & & & \\
\hline$S_{15}$ & $43 \rightarrow 53(0.57)$ & $\pi_{\mathrm{H}} \rightarrow \mathrm{Ryd}_{5}$ & 203 & & 0.0385 & \\
\hline$S_{16}$ & $42 \rightarrow 46(0.52)$ & $n_{\mathrm{H}-1} \rightarrow \mathrm{Ryd}_{2}$ & 199 & & 0.0518 & \\
\hline
\end{tabular}


发态反应动力学中具有重要意义. 水的溶剂效应对 不同类型的跃迁有不同程度的影响. 总体上, 在 SCIPCM 溶剂模型 ${ }^{[27]}$ 下, 溶剂化对 $\pi \rightarrow \pi^{*} 、 n \rightarrow \pi^{*} 、 \pi_{\mathrm{H}} \rightarrow$ $\mathrm{Ryd}_{3} 、 \pi_{\mathrm{H}} \rightarrow \mathrm{Ryd}_{4} 、 \pi_{\mathrm{H}} \rightarrow \mathrm{Ryd}_{5}$ 的跃迁能影响不大. 在 $A$ 带 吸收中, 溶剂效应使 $\pi \rightarrow \pi^{*}$ 跃迁变得更重要, 其振子 强度占整个 $A$ 带计算振子强度的 $95 \%$, 而 $\pi_{\mathrm{H}} \rightarrow \mathrm{Ryd}_{2}$ 跃迁不再起作用. 在 $B$ 带吸收中, 溶剂效应使 $\pi \rightarrow \pi^{*}$ 跃迁在计算振子强度中占据更大比重.

\section{4 共振拉曼光谱和激发态结构动力学}

经过强度校正和溶剂扣减后的9H-DMA 在水 和乙腈中的 $208.8 、 217.8 、 239.5 、 266.0 、 273.9$ 和 $282.4 \mathrm{~nm}$ 激发波长下的共振拉曼光谱示于图 4, 其 中 208.8 和 $217.8 \mathrm{~nm}$ 的共振拉曼光谱强度模式主要 反映 $B$ 吸收带电子跃迁时的动态结构信息, 而 266.0、273.9 和 $282.4 \mathrm{~nm}$ 共振拉曼光谱强度模式则 主要体现 $A$ 吸收带电子跃迁的动态结构信息. 图 4 可见, $A$ 和 $B$ 吸收带的共振拉曼光谱的强度模式有 很大差异, 说明它们激发态动态结构差别较大. 与 溶剂对紫外光谱的弱小影响一样, 溶剂对共振拉曼 光谱的影响不大, 表明水和乙腈对 FC 区域激发态 势能面的调控效应不明显.

图 5 示出 $9 \mathrm{H}-\mathrm{DMA}$ 在水溶液中的 $266.0 \mathrm{~nm}(A$ 带)和 $217.8 \mathrm{~nm}$ ( $B$ 带)共振拉曼光谱和它们的光谱指 认. 光谱中的绝大部分拉曼谱带可以分别被指认为

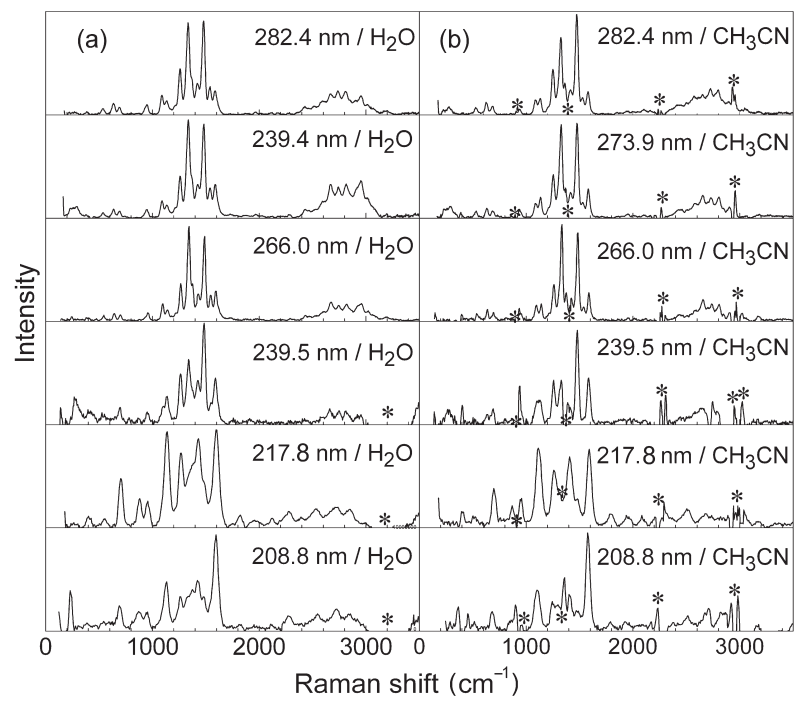

图 4 9H-DMA 分别在水(a)和乙腈(b)中的共振拉曼光谱

Fig.4 Overall view of resonance Raman spectra of 9H-DMA in water (a) and acetonitrile (b) solvents The spectra were obtained with the excitation wavelengths (in $\mathrm{nm}$ ) indicated next to each spectrum. The spectra have been intensity-corrected and solvent-subtracted. * Solvent subtraction artifacts are present.
15-17 个 FC 区域活性振动模的基频、泛频和合频. 我们注意到, 某些拉曼谱带可能由多条拉曼位移十 分相近的拉曼谱带组成, 但由于溶液相拉曼光谱有 限的分辨率, 图 5 仅列出了那些贡献最大的拉曼谱 带的指认. 由图 5 可见, $A$ 带和 $B$ 带共振拉曼光谱的 强度模式有着重大差别. 首先, $A$ 吸收带共振拉曼光 谱中对激发态动态结构起决定作用的是嘌呤环变 形伸缩 $+\mathrm{C} 8 \mathrm{H} / \mathrm{N} 9 \mathrm{H}$ 弯曲振动 $v_{23}$ 和五元环变形伸缩 + $\mathrm{C} 8 \mathrm{H}$ 弯曲振动 $v_{13}$, 其次是 $v_{26}$ 和 $v_{10}$, 它们的基频、泛频 和合频占据了共振拉曼光谱强度的绝大部分. 这说 明 ${ }^{1} \pi \pi^{*}$ 激发态动态结构变化主要沿着嘌呤环的伸缩 变形振动, $\mathrm{N} 9 \mathrm{H} / \mathrm{C} 8 \mathrm{H} / \mathrm{C} 2 \mathrm{H}$ 弯曲振动等反应坐标展 开, 并符合 $79 \%$ 的 $\pi_{\mathrm{H}} \rightarrow \pi_{\mathrm{L}}^{*}$ 跃迁和 $19 \%$ 的 $\pi_{\mathrm{H}} \rightarrow \mathrm{Ryd}_{2}$ 跃 迁的 $A$ 带吸收特征. 而 $B$ 吸收带共振拉曼光谱中对 激发态动态结构起决定作用的是 $v_{10}$ 和 $v_{29}$, 其次是 $v_{21}$ 、 $v_{26}$ 和 $v_{40}$ 等, 它们的基频、泛频和合频占据了共振拉 曼光谱强度的绝大部分.

特别值得关注的是, 原本在 $A$ 带共振拉曼光谱 中强度很弱的振动模式 $v_{29}$ (嘌呤环伸缩变形 + 二甲基
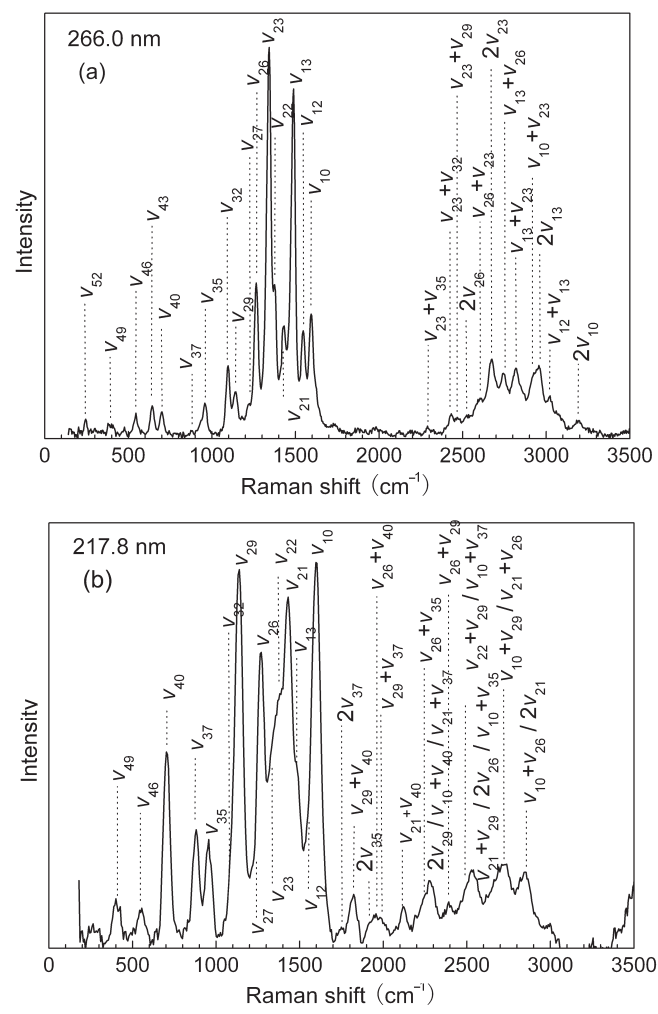

图 $59 H-D M A$ 在水溶液中的 $266.0 \mathrm{~nm}(\mathrm{a})$ 和 $217.8 \mathrm{~nm}(\mathrm{~b})$ 共振拉曼光谱指认

Fig.5 Tentative vibrational assignments of the $266 \mathrm{~nm}$ (a) and $217.8 \mathrm{~nm}$ (b) resonance Raman spectra of 9H-DMA in water

The spectra have been intensity-corrected and solvent-subtracted. 
氨基对称伸缩振动 $+\mathrm{C} 8 \mathrm{H}$ 弯曲振动)和强度中等的振 动模式 $v_{10}$ (嘌呤环伸缩变形 $+\mathrm{C} 6 \mathrm{~N} 10$ 伸缩振动 $+\mathrm{N} 9 \mathrm{H} /$ $\mathrm{C} 2 \mathrm{H}$ 弯曲振动 $+\mathrm{CH}_{3}$ 剪式振动), 在 $B$ 带光谱中成为 强度最大的振动模式. 原本在 $A$ 带共振拉曼光谱中 弱强度的振动模式 $v_{40}$ (嘌呤环伸缩变形)和极弱强度 的 $v_{37}$ (嘌呤环伸缩变形 + 二甲基氨基对称伸缩振动), 连同中等强度的振动模式 $v_{26}$ (嘌呤环伸缩变形 + 二甲 基氨基不对称伸缩振动 $+\mathrm{C} 8 \mathrm{H} / \mathrm{N} 9 \mathrm{H}$ 弯曲振动 $+\mathrm{CH}_{3}$ rock $)$ 和 $v_{21}(\mathrm{~N} 9 \mathrm{H} / \mathrm{C} 2 \mathrm{H}$ 弯曲+嘌呤环伸缩变形), 成为 $B$ 带光谱中相当强的振动模. 这六个振动模决定了 9H-DMA 激发态动态结构变化. 相反, 原本在 $A$ 带共 振拉曼光谱中强度最大的振动模 $v_{23}$ (主特征为嘌呤 环伸缩变形 $+\mathrm{C} 8 \mathrm{H} / \mathrm{N} 9 \mathrm{H}$ 弯曲振动) 和 $v_{13}$ (嘌呤环伸缩 变形 $+\mathrm{C} 8 \mathrm{H}$ 弯曲振动) 在 $B$ 带共振拉曼光谱中成为强 度很弱的振动模, 在该激发态动态结构变化中作用 不大. 总体上, $A$ 带和 $B$ 带共振拉曼光谱的这种基频 强度的变化与它们各自的泛频和合频的强度变化 规律基本一致, 说明 $B$ 带共振增强效应对 $A$ 带共振 拉曼光谱强度的贡献不大. 因此, 用它们各自的强 度模式来提取 $A$ 和 $B$ 带激发态动态结构信息是安全 的.

$A$ 带共振拉曼光谱中 $v_{26}$ 和 $v_{12}$ 的强度来源令人关 注. 在 $A$ 带电子跃迁的五个跃迁轨道中, 除了轨道 42 ( $n$ 轨道) 外, 其它 $43 、 44 、 45$ 和 46 轨道之间的电子 跃迁难以引起二甲氨基不对称伸缩振动 $v_{26}$ 和 $\mathrm{C}_{6} \mathrm{~N}_{10}$ 伸缩振动 $v_{12}$. 尽管 $A$ 带共振拉曼光谱中 $v_{26}$ 以及 $v_{10}$ 、 $v_{21} 、 \mathrm{~V}_{22} 、 \mathrm{~V}_{29}$ 等的基频强度有可能来自于 $\mathrm{V}$ 带预共振 增强效应的贡献, 因为这些振动模在 $B$ 带共振拉曼 光谱中具有很大强度, 但由于 $B$ 带的预共振增强效 应一般仅对 $A$ 带共振拉曼光谱中的基频有贡献, 而 我们注意到在 $A$ 带共振拉曼光谱中出现了相当强的 合频 $v_{13}+v_{26} 、 v_{12}+v_{13}$ 和明显的泛频 $2 v_{26} 、 2 v_{12}$. 这些合频 和泛频的出现说明 $v_{26}$ 和 $v_{12}$ 的拉曼强度来自于 $A$ 带电 子跃迁本身. 因此, 结合最近关于 FC 区域势能面交 叉的共振拉曼光谱强度分析研究 ${ }^{[28]}$ 和 Marian 等 ${ }^{[26]}$ 关 于腺嘌呤激发态势能面交叉的理论计算结果, 我们 认为它们的出现与 ${ }^{1} n_{\mathrm{H}-1} \pi_{\mathrm{L}}^{*}$ 态, 或 ${ }^{1} n \pi /{ }^{1} \pi \pi$ 势能面雉型交 叉有关. 我们注意到, Schwalb 和 Temps ${ }^{[20]}$ 近期对 DMA 的 $A$ 带荧光动力学机制开展了飞秒时间分辨 荧光上转换光谱实验研究. 通过考察 $A$ 带苂光的上 升时间随泵浦激光波长的变化情况, 发现 $A$ 带苂光 的产生几乎是即刻完成的和无能垒的过程, 并把 $A$ 苂光归属为 ${ }^{1} n \pi^{*}$ 态的发射, 这与本文揭示的 “DMA
存在 Franck-Condon 区域 ${ }^{1} n \pi /{ }^{\prime} \pi$ 势能面雉型交叉” 结 论相一致.

相对于 $A$ 带共振拉曼光谱, $v_{21}$ 振动模(主特征为 $\mathrm{N} 9 \mathrm{H} / \mathrm{C} 2 \mathrm{H}$ 弯曲) 在 $B$ 带共振拉曼光谱中的强度大大 增强. $v_{21}$ 的泛频及其与 $v_{10}, v_{29}, v_{26}, v_{37}, v_{40}$ 合频的出现 说明 $v_{21}$ 的共振拉曼活性来自于 $B$ 带电子跃迁本身, 而非高激发态的预共振效应. 由于 $v_{21}$ 反应坐标的激 活与弥散轨道 $\mathrm{Ryd}_{3}$ 的 $\sigma_{\mathrm{N} 9 \mathrm{H}}^{*}$ 特征相关, 但难以直接从 $\pi_{\mathrm{H}} \rightarrow \mathrm{Ryd}_{3}$ 跃迁中产生, 因为其振子强度太小 $(f=$ 0.0034), 也难以从表 2 中其它轨道的电子跃迁中产 生, 因为轨道特征不符合. 我们注意到, CASPT2// CASSCF 计算研究显示, 腺嘌呤在沿 N9-H 反应坐标 上存在 $S_{0} / \pi \sigma_{\mathrm{N} 9 H}^{*}$ 的雉形交叉 ${ }^{[29]}$. 超快时间分辨光谱实 验揭示 ${ }^{[9]}$, 由于 ${ }^{1} \pi^{*} /{ }^{1} \pi \sigma_{\mathrm{N} 9 H}^{*}$ 雉形交叉机制的存在, $B$ 带电 子激发导致 9H-DMA 的 N9-H 解离. 因此, $B$ 带共振 拉曼光谱中 $v_{21}$ 振动模强度大大增强, 一种合理的解 释是, 在 Franck-Condon 区域存在 ${ }^{1} \pi \pi^{*}$ (或 ${ }^{1} \pi \mathrm{Ryd}$ )/ ${ }^{1} \pi_{\mathrm{H}} \mathrm{Ryd}_{3}$ 雉形交叉.

\section{4 结 论}

采用共振拉曼光谱技术和密度泛函理论方法 研究了 6- $N, N$-二甲基腺嘌呤(DMA)的 $A$ 带和 $B$ 带电 子激发、Franck-Condon 区域结构动力学和势能面非 绝热交叉, 得出如下结论:

(1) $9 \mathrm{H}$-异构体非平面重叠构象和 7H-异构体非 平面交叉构象是 DMA 基态势能面上两个能量最低 点结构. 在 B3LYP/6-31++g $(d f, p d)$ 计算水平下, $9 \mathrm{H}-$ 异构体和 $7 \mathrm{H}$-异构体的能差为 $37.38 \mathrm{~kJ} \cdot \mathrm{mol}^{-1}$ (零点 能校正后为 $\left.34.40 \mathrm{~kJ} \cdot \mathrm{mol}^{-1}\right)$.

(2) $\pi_{\mathrm{H}} \rightarrow \pi_{\mathrm{L}}^{*}$ 跃迁是 $A$ 带吸收的主体, 其振子强度 约占整个 $A$ 带吸收的 79\%. 由弥散轨道参与的 $n \rightarrow$ $\operatorname{Ryd}$ 和 $\pi_{\mathrm{H}} \rightarrow \operatorname{Ryd}$ 跃迁在 $B$ 带跃迁中扮演重要角色, 其 振子强度约占 $B$ 带吸收的 $62 \%$, 而在 $A$ 带吸收中占 主导的 $\pi \rightarrow \pi^{*}$ 跃迁的振子强度在 $B$ 带吸收中仅占 $33 \%$.

(3) 嘌呤环变形伸缩 $+\mathrm{C} 8 \mathrm{H} / \mathrm{N} 9 \mathrm{H}$ 弯曲振动 $v_{23}$ 和 五元环变形伸缩 $+\mathrm{C} 8 \mathrm{H}$ 弯曲振动 $v_{13}$ 的基频、泛频和 合频占据了 $A$ 带共振拉曼光谱强度的绝大部分, 说 明 ${ }^{1} \pi_{\mathrm{H}} \pi_{\mathrm{L}}^{*}$ 激发态结构动力学主要沿嘌呤环的变形伸 缩振动、 $\mathrm{N}_{9} \mathrm{H} / \mathrm{C} 8 \mathrm{H} / \mathrm{C} 2 \mathrm{H}$ 弯曲振动等反应坐标展 开, 而 $v_{10} 、 v_{29}, v_{21} 、 v_{26}$ 和 $v_{40}$ 的基频、泛频和合频占据了 $B$ 带共振拉曼光谱强度的主体部分, 它们决定了 $B$ 带激发态的结构动力学. 
(4) $A$ 带共振拉曼光谱中 $v_{26}$ 和 $v_{12}$ 的出现与 ${ }^{1} n \pi /$ ${ }^{1} \pi \pi$ 势能面锥型交叉有关. $B$ 带共振拉曼光谱中 $v_{21}$ 的激活与 ${ }^{1} \pi \pi^{*} / 1 \pi \sigma^{*}$ NH 势能面锥型交叉相关. FranckCondon 区域 ${ }^{1} n \pi /^{1} \pi \pi$ 势能面雉型交叉的结论, 得到了 近期荧光上转换实验结果的支持. 这一事实表明, 从势能面非绝热交叉的视角, 通过深入分析核酸碱 基及其衍生物的共振拉曼光谱强度模式, 可以有效 探索分子激发结构动力学、识别和获取激发态势能 面绝热和非绝热交叉动力学信息, 为核酸碱基激发 态超快动力学研究提供新信息.

\section{References}

1 Crespo-Hemández, C. E.; Cohen, B.; Hare, P. M.; Kohler, B. Chem. Rev., 2004, 104: 1977

2 Sobolewski, A. L.; Domcke, W.; Dedonder-Lardeux, C.; Jouvet, C. Phys. Chem. Chem. Phys., 2002, 4: 1093

3 Perun, S.; Sobolewski, A. L.; Domcke,W. J. Am. Chem. Soc., 2005, 127: 6257

4 Perun, S.; Sobolewski, A. L.; Domcke, W. Chem. Phys., 2005, 313: 107

5 Kang, H.; Jung, B.; Kim, S. K. J. Chem. Phys., 2003, 118: 6717

6 Canuel, C.; Mons, M.; Piuzzi, F.; Tardivel, B.; Dimicoli, I.; Elhanine, M. J. Chem. Phys., 2005, 122: 074306

7 Samoylova, E.; Lippert, H.; Ullrich, S.; Hertel, I. V.; Radloff, W.; Schultz, T. J. Am. Chem. Soc., 2005, 127: 1782

8 Wells, K. L.; Roberts, G. M.; Stavros, V. G. Chem. Phys. Lett., 2007, 446: 20

9 Wells, K. L.; Hadden, D. J.; Nix, M. G. D.; Stavros, V. G. J. Phys. Chem. Lett., 2010, 1: 993

10 Zierhut, M.; Roth, W.; Fischer, I. Phys. Chem. Chem. Phys., 2004, 6: 5178

11 Satzger, H.; Townsend, D.; Zgierski, M. Z.; Patchovskii, S.; Ullrich, S.; Stolow, A. Proc. Natl. Acad. Sci. U. S. A., 2006, 103:
10196

12 Nix, M. G. D.; Devine, A. L.; Cronin, B.; Ashfold, M. N. R. J. Chem. Phys., 2007, 126: 124312

13 Ullrich, S.; Schultz, T.; Zgierski, M. Z.; Stolow, A. Phys. Chem. Chem. Phys., 2004, 6: 2796

14 Ullrich, S.; Schultz, T.; Zgierski, M. Z.; Stolow, A. J. Am. Chem. Soc., 2004, 126: 2262

15 Ritze, H. H.; Lippert, H.; Samoylova, E.; Smith, V. R.; Hertel, I. V.; Radloff, W.; Schultz, T. J. Chem. Phys., 2005, 122: 224320

16 Longworth, J. W.; Rahn, R. O.; Shulman, R. G. J. Chem. Phys., 1966, 45: 2930

17 Wilson, R. W.; Callis, P. R. Photochem. Photobiol., 1974, 20: 345

18 Eastman, J. W. Ber. Bunsen-Ges. Phys. Chem., 1969, 75: 407

19 Parusel, A. B. J.; Rettig, W.; Rotkiewicz, K. J. Phys. Chem. A, 2002, 106: 2293

20 Schwalb, N. K.; Temps, F. Phys. Chem. Chem. Phys., 2006, 8: 5229

21 Li, S. P.; Wu, G. M.; Zheng, X. M. Chem. J. Chin. Univ., 2004, 25: 1495 [李少鹏, 吴光明, 郑旭明. 高等学校化学学报, 2004, 25 : 1495]

22 Myer, A. B.; Li, B.; Ci, X. J. Chem. Phys., 1988, 89: 1876

23 Frisch, M. J.; Trucks, G. W.; Schlegel, H. B.; et al. Gaussian 03, Revision B.02. Pittsburgh, PA: Gaussian Inc., 2003

24 Sadlej-Sosnowska, N.; Krygowski, T. M. Chem. Phys. Lett., 2009, 476: 191

25 Rauhut, G.; Pulay, P. J. Phys. Chem., 1995, 99: 3091

26 Marian, C. M. J. Chem. Phys., 2005, 122: 104314

27 Foresman, J. B.; Keith, T. A.; Wiberg, K. B.; Snoonian, J.; Frisch, M. J. J. Phys. Chem., 1996, 100: 16098

28 Wu, X. F.; Zheng, X.; Wang, H. G.; Zhao, Y. Y.; Guan, X. G.; Phillips, D. L.; Chen, X. B.; Fang, W. H. J. Chem. Phys., 2010, 133: 134507

29 Conti, I.; Garavelli, M.; Orlandi, G. J. Am. Chem. Soc., 2009, 131: 16118 